\title{
Parapapillary Intrachoroidal Cavitation in Glaucoma: Association with Choroidal Microvasculature Dropout
}

\author{
Jounghan Kim, Jiah Kim, Eun Ji Lee, Tae-Woo Kim \\ Department of Ophthalmology, Seoul National University Bundang Hospital, Seoul National University College of Medicine, \\ Seongnam, Korea
}

\begin{abstract}
Purpose: To characterize the choroidal microvasculature in glaucomatous eyes with parapapillary intrachoroidal cavitation (PICC) using optical coherence tomography angiography (OCTA) and its association with parapapillary choroidal microvasculature dropout (MvD).

Methods: This study included 47 glaucomatous eyes with PICC, as identified by color fundus photography and optical coherence tomography scanning of the optic nerve head area. Peripapillary choroidal microvasculature was evaluated on en-face OCTA images. Choroidal MvD was defined as a focal sectoral capillary dropout with no visible microvascular network.

Results: PICC was visible as a well-demarcated area with homogeneously reduced vessel density in en-face OCTA images of the choroidal layer. MvD was detected in 42 eyes (89.4\%). Although located in the juxtapapillary area adjacent to the PICC, MvD was confined to the area of parapapillary atrophy. MvD observed in OCTA en-face images was distinguished from the area of PICC by the absence of vascular signal. Of the 50 PICCs, 49 (98.0\%) had hemifield visual field defects at the location corresponding to the hemispheric location of PICC.

Conclusions: PICC was found to have a characteristic microvascular feature in choroidal en-face OCTA images, and to be topographically associated with glaucomatous visual field defect. PICC was frequently accompanied by MvD and was located adjacent to the area of MvD, suggesting that PICC and MvD have similar pathogenesis.
\end{abstract}

Key Words: Choroidal microvasculature dropout, Glaucoma, Optical coherence tomography angiography, Peripapillary intrachoroidal cavitation

Peripapillary intrachoroidal cavitation (PICC), which has been described as a hypovascular cavity within the peripapillary choroidal layer, is exclusively found in highly

Received: October 22, 2020 Final revision: November 19, 2020 Accepted: November 19, 2020

Corresponding Author: Eun Ji Lee, MD. Department of Ophthalmology, Seoul National University Bundang Hospital, 82 Gumi-ro 173beon-gil, Bundang-gu, Seongnam 13620, Korea. Tel: 82-31-787-7378, Fax: 82-31787-4057, E-mail: opticdisc@snubh.org myopic eyes [1-10]. PICC appears as a yellowish-orange lesion located adjacent to the optic disc in color fundus photographs [1]. The development of the choroidal cavity is thought to involve posterior excursion of the sclera during the course of myopic expansion of globe [2-5], or the presence of structurally weaker peripapillary border tissue [9,11]. Glaucomatous visual field (VF) defects have been frequently detected in eyes with PICC $[3,4,11]$. Tilting of the optic disc is thought to induce structural distortion of the retinal nerve fiber layer (RNFL), resulting in subse- 
quent mechanical damage to the RNFL $[3,4]$.

Optical coherence tomography (OCT) angiography (OCTA) has identified a localized microvasculature dropout $(\mathrm{MvD})$ in the peripapillary choroidal layer of glaucoma patients [12,13], with the location of the MvD correlating with the location of glaucomatous damage $[13,14]$. MvD has been reported in $76 \%$ to $78 \%$ of glaucomatous eyes with myopia [15,16], with this MvD being associated with longer axial length and larger parapapillary $\gamma$-zone. These findings indicated that $\mathrm{MvD}$ associated with myopia may develop by stretching of the microvessels in the scleral flange during myopic globe expansion $[15,16]$.

The peripapillary choroidal microvasculature is of particular interest in glaucoma because it is supplied by the short posterior ciliary arteries, which also perfuse deep optic nerve head tissues. Both PICC and MvD involve changes in deep parapapillary structures, including the choroid. Given the relatively high frequency of PICC and $\mathrm{MvD}$ in highly myopic eyes, and their potential association with glaucoma, the present study examined whether there are differences in the microvasculature between PICC and MvD.

\section{Materials and Methods}

The subjects of this study consisted of patients with primary open-angle glaucoma (POAG) who were participating in the Investigating Glaucoma Progression Study, an ongoing prospective study being performed at the Glaucoma Clinic of Seoul National University Bundang Hospital, Seongnam, Korea. The protocol of this study was approved by the institutional review board of Seoul National University Bundang Hospital (B-2009-634-115) and conformed to the tenets of the Declaration of Helsinki. All study subjects provided written informed consent to participate.

\section{Study subjects}

The database of patients with POAG who were enrolled in the Investigating Glaucoma Progression Study was reviewed to identify those patients who had undergone both swept-source OCTA and OCT scanning of the optic disc and peripapillary area. All subjects underwent complete ophthalmic examinations, including visual acuity assess- ment, refraction, slit-lamp biomicroscopy, gonioscopy, Goldmann applanation tonometry, and dilated stereoscopic examination of the optic disc. Central corneal thickness was measured by Orbscan II (Bausch \& Lomb Surgical, Rochester, NY, USA) and axial length by IOL Master version 5 (Carl Zeiss Meditec, Dublin, CA, USA). Stereo disc photography was performed with an EOS D60 digital camera (Canon, Utsunomiya, Japan), the circumpapillary RNFL was measured using spectral-domain OCT (Spectralis OCT; Heidelberg Engineering, Heidelberg, Germany), optic discs were scanned using swept-source OCTA (DRI-OCT Triton; Topcon, Tokyo, Japan), and standard automated perimetry was performed using a 24-2 Swedish interactive threshold algorithm and a Humphrey Field Analyzer II 750 (Carl Zeiss Meditec).

Eyes were included if they had a PICC, as confirmed by both color fundus photography and OCT scanning of the optic disc (Fig. 1A-1E). A hypovascular cavity in the choroidal layer on OCT B-scan images that corresponded to a yellowish-orange parapapillary lesion on fundus photography was deemed a PICC. Eyes with a history of ocular surgery other than cataract extraction and glaucoma surgery and eyes with other intraocular diseases (e.g., age-related macular degeneration, pathologic myopia, diabetic retinopathy, or retinal vessel occlusion) were excluded, as were eyes of patients with neurological diseases (e.g., pituitary tumors) that could cause VF defects and eyes with a history of ocular trauma or uveitis.

POAG was defined as an open iridocorneal angle, abnormal glaucomatous optic disc (diffuse or focal thinning of the neuroretinal rim [17] or splinter hemorrhage), and associated VF defects without other ocular diseases or conditions that might cause VF abnormalities. A glaucomatous VF defect was defined as (1) values outside the normal limits on glaucoma hemifield tests; (2) three abnormal points, each with a probability $<5 \%$ of being normal, and one point with a pattern deviation $<1 \%$; or (3) a pattern standard deviation $<5 \%$. These VF defects were confirmed in two consecutive reliable tests with fixation loss rates $\leq 20 \%$ and false-positive and false-negative error rates $\leq 25 \%$. Untreated intraocular pressure was defined as the mean of at least two measurements prior to the start of intraocular pressure-lowering treatment. 


\section{Swept-source OCT and OCTA}

The optic nerve and peripapillary area were imaged using a commercially available swept-source OCTA device (DRI OCT Triton, Topcon), with a central wavelength of $1,050 \mathrm{~nm}$, an acquisition speed of 100,000 A scans per second, and axial and transversal resolutions in tissue of 7 and $20 \mu \mathrm{m}$, respectively. Scans were taken from $4.5 \mathrm{~mm} \times 4.5$ $\mathrm{mm}$ cubes, with each cube consisting of 320 clusters of four repeated B scans centered on the optic disc. En-face projections of volumetric scans allowed visualization of structural and vascular details within segmented retinal and choroidal layers.

A PICC was defined as a hypovascular space in the choroidal layer on B-scan images, corresponding to yellowish-orange lesions on color fundus images (Fig. 1). The hemispheric locations of PICCs relative to the foveal-disc

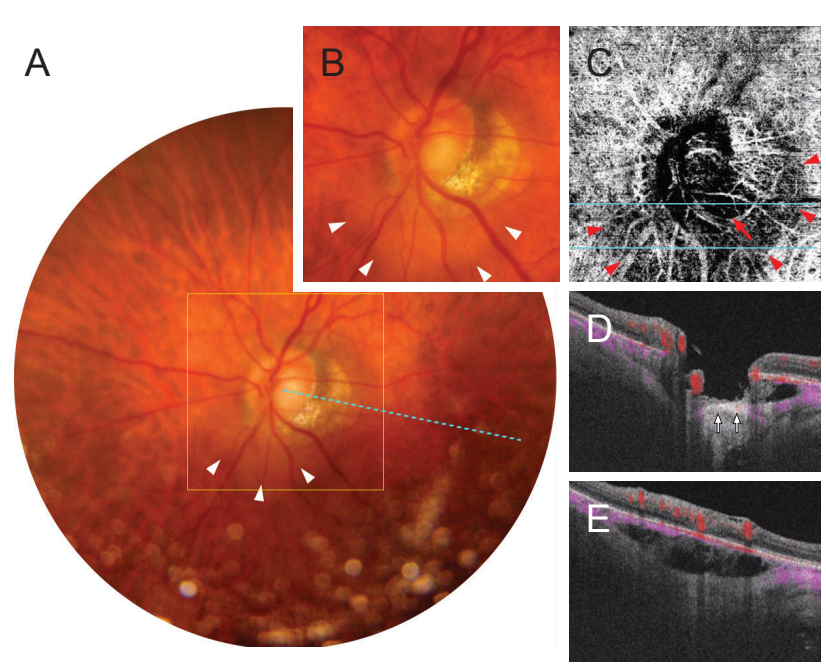

Fig. 1. Identification of parapapillary intrachoroidal cavitation (PICC) and parapapillary choroidal microvasculature dropout (MvD). (A) Color photograph showing a PICC shown as a yellowish-orange lesion around the optic nerve head (arrowheads). Dashed line indicates foveal-disc axis. (B) Enlarged image of the yellow-squared area in (A). (C) En-face optical coherence tomography angiography (OCTA) image obtained in the choroidal layer. (D,E) B-scan images obtained at the location indicated with light blue lines in (B). (C) In the choroidal OCTA image, PICC was observed as a large hypovascular area (arrowheads), which was distinctive from the region of $\mathrm{MvD}$ (arrow) by differed microvascular density. (D,E) The margin of the PICC was corresponded to the choroidal cavity shown in the B-scan images. Note that the edge of the cavitation is more evident in the OCTA image (C) than it is in the color fundus photo $(\mathrm{A}, \mathrm{B})$. In B-scan images $(\mathrm{D}, \mathrm{E})$, a large choroidal cavity is identified under the Bruch's membrane. Note that the PICC is accompanied by vascular signals from choriocapillaris, while $\mathrm{MvD}$ is characterized by absence of vascular signal (arrows, D). axis were determined and recorded as superior or inferior.

The peripapillary microvasculature was evaluated in enface images of the peripapillary choroidal layer, generated based on automated layer segmentation by the OCT instrument software. The en-face images of the choroidal layer were derived from en-face slabs extending from the retinal pigment epithelium to $390 \mu \mathrm{m}$ below Bruch's membrane, which included the full thickness of the choroid and the inner scleral surface.

MvD was defined as a focal sectoral capillary dropout with no visible microvascular network identified on deep-layer en-face images (Fig. 1) [13,14,18]. A circumferential width of the area with capillary dropout greater than one-half clock hour of the disc circumference was considered a disruption of the microvascular network and deemed an MvD. The hemispheric locations of MvDs relative to the foveal-disc axis were determined and recorded as superior or inferior. MvDs were determined by two independent observers (JK and JAK) blinded to the clinical characteristics of the subjects. Disagreements between these observers were resolved by a third observer (EJL). All OCT B scan images had image quality scores $\geq 30$, as recommended by the manufacturer. Eyes with poor OCTA images (i.e., blurred images that hampered the delineation of the MvD) were excluded.

\section{Statistical analysis}

Except where stated otherwise, all data were presented as mean \pm standard deviation. Interobserver agreements for determining the presence of an $\mathrm{MvD}$ and topographic correlations between the hemispheric locations of PICC and hemifield locations of VF defects were assessed using kappa statistics ( $\mathrm{k}$-value). All statistical analyses were performed using the IBM SPSS Statistics ver. 22.0 (IBM Corp., Armonk, NY, USA), with $p$-values $<0.05$ defined as statistically significant.

\section{Results}

The study initially included 52 eyes of 43 POAG patients with PICC. Of these, five eyes of four patients were excluded because of the poor quality of their OCTA images. Thus, this study included 47 eyes of 39 POAG patients. The demographic and clinical characteristics of these par- 
ticipants are shown in Table 1. The mean axial length of included eyes was $27.42 \pm 1.40 \mathrm{~mm}$ (range, 25.51 to 30.05 $\mathrm{mm})$.

Table 1. Demographics of the participants

\begin{tabular}{|c|c|}
\hline Variable & Value \\
\hline Age $(y r)^{*}$ & $49.7 \pm 12.6$ \\
\hline Sex (male : female) ${ }^{*}$ & $19: 20$ \\
\hline Presence of MvD & $42(89.4)$ \\
\hline Untreated IOP & $14.1 \pm 2.3$ \\
\hline IOP at OCTA & $12.4 \pm 2.7$ \\
\hline Axial length (mm) & $27.4 \pm 1.4$ \\
\hline Corneal thickness $(\mu \mathrm{m})$ & $544 \pm 76$ \\
\hline Global RNFL thickness $(\mu \mathrm{m})$ & $77.2 \pm 12.1$ \\
\hline Visual field MD (dB) & $-4.6 \pm 3.2$ \\
\hline Visual field PSD (dB) & $4.6 \pm 3.3$ \\
\hline
\end{tabular}

Values are presented as mean \pm standard deviation, number, number (\%).

$\mathrm{MvD}=$ microvasculature dropout; IOP = intraocular pressure; OCTA $=$ optical coherence tomography angiography; RNFL = retinal nerve fiber layer; $\mathrm{MD}=$ mean deviation; $\mathrm{PSD}=$ pattern standard deviation.

"These variables are based on the data from 39 individual participants. Others are based on the data from 47 eyes.
PICC was observed in the inferior hemisphere of 43 eyes, the superior hemisphere of one eye, and both superior and inferior hemispheres of three eyes, resulting in a total of 50 PICCs. En-face choroidal OCTA images identified PICCs as well-demarcated homogeneous areas of diffusely reduced vessel density, corresponding to the yellow-orange lesions observed on fundus photographs and hyporeflective choroidal cavities on OCT B-scans (Fig. 1, 2A-2G). While the cavity was devoid of vascular signals from the choroid, choriocapillaris signal was present in the area of PICC. The base of each PICC adjoined the optic disc border, and the distal border extended outside the PPA.

MvD was observed in 42 eyes (89.4\%) in the juxtapapillary area, with all being proximally adjacent to the PICC. Interobserver agreement in detecting the presence of $\mathrm{MvD}$ was excellent $(\kappa=0.953)$. Table 2 shows the hemispheric locations of PICCs and MvDs. There was a substantial agreement [19] between the hemispheric locations of PICC and $\operatorname{MvD}(\kappa=0.791)$. MvDs could be clearly distinguished from PICCs on choroidal OCTA images by the complete loss of vascular signals in the former (Fig. 1, 2). MvDs appeared as juxtapapillary focal dropout of microvessels and did not extend beyond PPA areas.

Of the 50 PICCs, 49 (98.0\%) were accompanied by
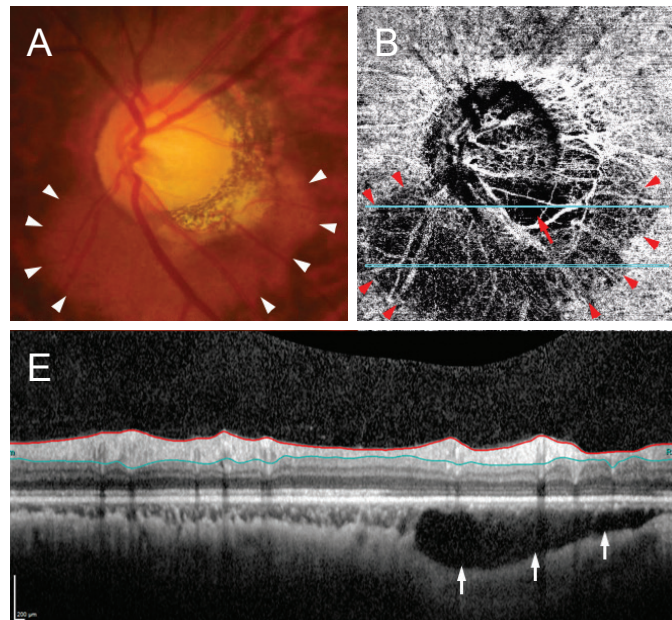

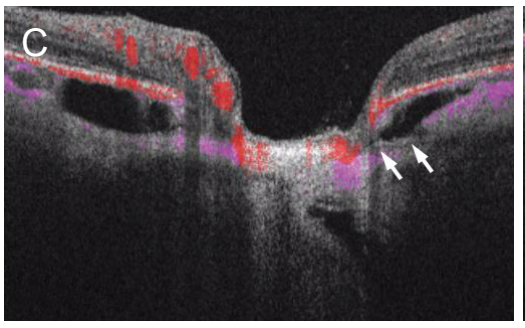

$\mathrm{F}$

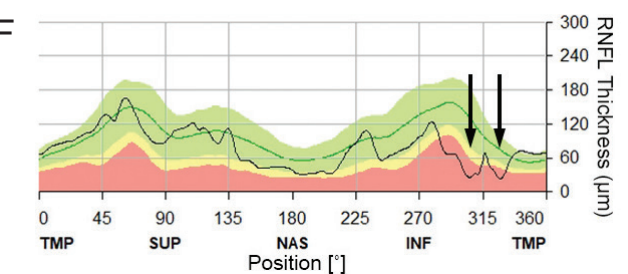

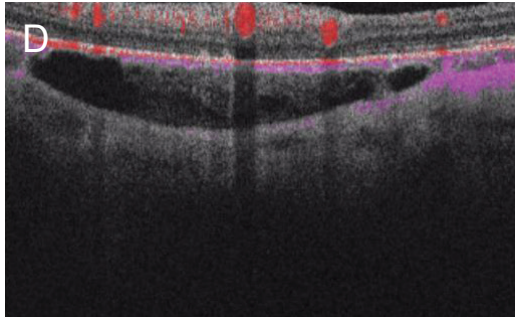

G

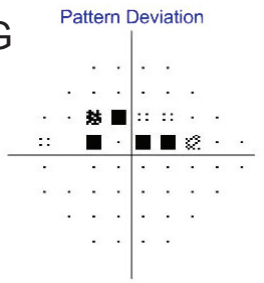

Fig. 2. A glaucomatous eye with a parapapillary intrachoroidal cavitation (PICC). (A) Color photograph showing a PICC along the inferior aspect of the optic nerve (arrowheads). (B) En-face optical coherence tomography angiography image obtained in the choroidal layer. PICC is observed as a large hypovascular area (arrowheads), which was distinctive from the region of microvasculature dropout (MvD, arrow) by differed microvascular density. (C,D) B-scan images obtained at the location indicated with blue lines in (B). A large choroidal cavity is identified under the Bruch's membrane. Some vascular signals from choriocapillaris are identified in the PICC (C,D), while MvD is characterized by absence of vascular signal (arrows, C). (E) Peripapillary B-scan image showing a cavity in the inferior parapapillary portion (arrows). (F) optical coherence tomography measurement of peripapillary retinal nerve fiber layer (RNFL) thickness. (G) Pattern deviation plot of standard automated perimetry. Note that the locations of decreased RNFL thickness (F, arrows) and hemifield visual field defect $(\mathrm{G})$ are corresponded to the location of the PICC (A, B, arrowheads) and MvD (B, arrow). TMP = temporal; SUP = superior; $\mathrm{NAS}=$ nasal; INF $=$ inferior. 
Table 2. Hemispheric location of PICC and MvD

\begin{tabular}{lccccc}
\hline \multirow{2}{*}{ Hemispheric location of PICC } & \multicolumn{3}{c}{ Hemispheric location of MvD } & \multirow{2}{*}{ Total } \\
\cline { 2 - 5 } & Inferior & Superior & Both & Absent & 43 \\
Inferior & 39 & 0 & 0 & 0 & 1 \\
Superior & 0 & 1 & 0 & 1 & 3 \\
Both & 0 & 1 & 1 & 5 & 47 \\
Total & 39 & 2 & 1 & 0 \\
\hline
\end{tabular}

$\mathrm{PICC}=$ parapapillary intrachoroidal cavitation; $\mathrm{MvD}=$ microvasculature dropout.

Table 3. Hemispheric location of PICC and hemifield location of visual field defect

\begin{tabular}{lcccc}
\hline \multirow{2}{*}{ Hemispheric location of PICC } & \multicolumn{3}{c}{ Hemifield location visual field defect } & \multirow{2}{*}{ Total } \\
\cline { 2 - 5 } & Inferior & Superior & Both & 12 \\
Inferior & 1 & 30 & 0 & 43 \\
Superior & 1 & 0 & 3 & 3 \\
Both & 0 & 30 & 15 & 47 \\
Sum & 2 & 0 & 3 \\
\hline
\end{tabular}

$\mathrm{PICC}=$ parapapillary intrachoroidal cavitation.

hemifield VF defects at the corresponding hemispheric location (Table 3 and Fig. 2). Kappa statistics revealed a topographic agreement between the hemispheric locations of PICCs and hemifield locations of VF defects $(\kappa=0.696)$.

\section{Discussion}

The present study described the microvascular characteristics of PICCs using en-face OCTA images of the choroidal layer in patients with POAG, in association with the MvD. Almost $90 \%$ of eyes with PICC also had MvD in the area proximal to the PICC. The microvascular features of PICCs on choroidal en-face OCTA images were distinct from those of MvD, as shown by differences in microvascular density. PICCs appeared as larger hypovascular areas, whereas MvD showed focal absence of vascular signal, indicating total dropout of microvessels.

The different appearance of the PICC and MvD in enface OCTA may reflect the structural difference of the microvessels within the PICC and MvD. Some choriocapillaris signal could be found at the location of PICC, despite of the cavity devoid of choroidal vasculature (Fig. 1E, Fig. 2D). However, MvD was characterized by absence of vas- cular signal indicating a total dropout of microvessels.

PICCs have been mainly detected in highly myopic eyes [1-10], but they have also been observed in eyes with low myopia and non-myopic eyes [11]. PICC formation may result from changes in the globe associated with progressive myopia [2-5]. Alternatively, PICCs may be induced by structurally weaker peripapillary border tissue prone to disruption [9,11]. Because PICC is exclusively found in the inferior location $[1,3]$, it has been suggested that it is an incomplete form of choroidal coloboma [1], or its location is affected by the gravitation of fluids [11]. Whichever the case is, the contortions of deep retinal structures associated with the formation of a PICC may result in structural distortion of the RNFL, eventually leading to mechanical damage to the RNFL [3,4]. Studies have shown that PICC is often accompanied by glaucomatous VF [3,4,11] or RNFL [11] damage. The present work, which included POAG patients with moderate to high myopia, showed that the location of PICC correlated topographically with the hemifield VF defect.

In the present study, almost $90 \%$ of the PICC was accompanied by MvD. Although the pathogenesis of MvD is unclear, peripapillary structural changes associated with myopia have been attributed to the development of MvD in 
myopic POAG eyes $[15,16]$. The proximity of MvD and PICC suggests that their pathogenesis lies on a common spectrum. Distortion of the peripapillary structure that could induce the formation of PICC may also cause mechanical stretching of nearby tissues. Tensile stress of long duration in peripapillary tissues could damage the RNFL and microvessels within the scleral flange, as well as leading to the occlusion of microvessels. These changes are more likely to occur in regions with the greatest tensile stress, which are linked to the direction of distortion [20-23].

It is important to distinguish between MvDs observed in this study and those described in general populations of patients with POAG. MvDs have been reported to be pathogenically significant in eyes with glaucomatous optic neuropathy, including non-myopic POAG eyes [24-29]. In addition, MvD was found to independently predict future glaucoma progression [28,29], suggesting that MvD may not simply be a secondary by-product of glaucomatous damage. The microstructure underlying the MvD has been found to differ in eyes with and without optic disc tilt, indicating that the mechanism of MvD development depends on optic disc morphology [18]. Additional studies are required to determine the temporal relationships among $\mathrm{MvD}, \mathrm{PICC}$ and glaucomatous progression.

Our demonstration of OCTA characteristics of PICCs may help distinguish this condition from other pathologic conditions in the choroid requiring treatment. However, the clinical importance of PICC remains unclear, with this condition rarely complicated by vision threatening conditions, such as macular detachment [4]. Careful evaluation of complications may be required regularly. In addition, longer-term observational studies may clarify prognosis in glaucoma patients with PICC associated with MvD.

The present study had limitations. First, peripapillary OCTA protocol scans assessed only limited areas, resulting in large PICCs that could not be entirely covered. Second, the clinical diagnosis of glaucoma may be difficult in myopic eyes because of anomalous optic disc changes [4] and atypical VF changes [30]. Because the present study only included eyes definitively diagnosed with glaucoma, these findings may not be applicable to all highly myopic glaucoma eyes with PICC. Third, this study aimed to focus on the OCTA findings of the PICC and associated clinical characteristics in glaucoma, and so only glaucomatous eyes with PICC were included. Inclusion of the eyes without PICC and only having MvD could help to better under- stand the clinical characteristics of PICC in glaucoma. Clinical importance of PICC should be addressed by a longitudinal study.

In conclusion, the microvasculature of PICC in glaucomatous eyes was characterized by OCTA images in association with MvD. Our findings of its topographic correlation with glaucomatous VF defects and high prevalence of accompanying MvD suggest common pathogenic mechanisms involving PICC and MvD in patients with glaucoma. Additional studies are needed to determine the clinical importance of PICC in glaucoma.

\section{Conflict of Interest}

No potential conflict of interest relevant to this article was reported.

\section{Acknowledgements}

This work was supported by Seoul National University Bundang Hospital Research Fund (no. 02-2017-037), Seongnam and by a grant of Patient-Centered Clinical Research Coordinating Center funded by the Ministry of Health \& Welfare, Republic of Korea (grant no. HI19C0481, HC19C0276).

\section{References}

1. Freund KB, Ciardella AP, Yannuzzi LA, et al. Peripapillary detachment in pathologic myopia. Arch Ophthalmol 2003;121:197-204.

2. Toranzo J, Cohen SY, Erginay A, Gaudric A. Peripapillary intrachoroidal cavitation in myopia. Am J Ophthalmol 2005;140:731-2

3. Shimada N, Ohno-Matsui K, Yoshida T, et al. Characteristics of peripapillary detachment in pathologic myopia. Arch Ophthalmol 2006;124:46-52.

4. Shimada N, Ohno-Matsui K, Nishimuta A, et al. Peripapillary changes detected by optical coherence tomography in eyes with high myopia. Ophthalmology 2007;114:2070-6.

5. Wei YH, Yang CM, Chen MS, et al. Peripapillary intrachoroidal cavitation in high myopia: reappraisal. Eye (Lond) 2009;23:141-4. 
6. Tateno H, Takahashi K, Fukuchi T, et al. Choroidal schisis around the optic nerve in myopic eyes evaluated by optical coherence tomography. Jpn J Clin Ophthalmol 2005;59:32731.

7. Forte R, Pascotto F, Cennamo G, de Crecchio G. Evaluation of peripapillary detachment in pathologic myopia with en face optical coherence tomography. Eye (Long) 2008;22:158-61.

8. Fujiwara T, Imamura Y, Margolis R, et al. Enhanced depth imaging optical coherence tomography of the choroid in highly myopic eyes. Am J Ophthalmol 2009;148:445-50.

9. Spaide RF, Akiba M, Ohno-Matsui K. Evaluation of peripapillary intrachoroidal cavitation with swept source and enhanced depth imaging optical coherence tomography. Retina 2012;32:1037-44.

10. You QS, Peng XY, Chen CX, et al. Peripapillary intrachoroidal cavitations. The Beijing eye study. PLoS One 2013;8:e78743.

11. Yeh SI, Chang WC, $\mathrm{Wu} \mathrm{CH}$, et al. Characteristics of peripapillary choroidal cavitation detected by optical coherence tomography. Ophthalmology 2013;120:544-52.

12. Akagi T, Iida Y, Nakanishi H, et al. Microvascular density in glaucomatous eyes with hemifield visual field defects: an optical coherence tomography angiography study. Am J Ophthalmol 2016;168:237-49.

13. Suh MH, Zangwill LM, Manalastas PI, et al. Deep retinal layer microvasculature dropout detected by the optical coherence tomography angiography in glaucoma. Ophthalmology 2016;123:2509-18.

14. Lee EJ, Lee KM, Lee SH, Kim TW. Parapapillary choroidal microvasculature dropout in glaucoma: a comparison between optical coherence tomography angiography and indocyanine green angiography. Ophthalmology 2017;124:120917.

15. Suh MH, Zangwill LM, Manalastas PI, et al. Deep-layer microvasculature dropout by optical coherence tomography angiography and microstructure of parapapillary atrophy. Invest Ophthalmol Vis Sci 2018;59:1995-2004.

16. Lee EJ, Kim TW, Kim JA, Kim JA. Parapapillary deep-layer microvasculature dropout in primary open-angle glaucoma eyes with a parapapillary $\gamma$-zone. Invest Ophthalmol Vis Sci 2017;58:5673-80.

17. Lopilly Park HY, Lee NY, Choi JA, Park CK. Measurement of scleral thickness using swept-source optical coherence tomography in patients with open-angle glaucoma and myopia. Am J Ophthalmol 2014;157:876-84.
18. Lee EJ, Kim TW, Lee SH, Kim JA. Underlying microstructure of parapapillary deep-layer capillary dropout identified by optical coherence tomography angiography. Invest Ophthalmol Vis Sci 2017;58:1621-7.

19. Landis JR, Koch GG. The measurement of observer agreement for categorical data. Biometrics 1977;33:159-74.

20. Lee KM, Lee EJ, Kim TW. Lamina cribrosa configuration in tilted optic discs with different tilt axes: a new hypothesis regarding optic disc tilt and torsion. Invest Ophthalmol Vis Sci 2015;56:2958-67.

21. Park HY, Lee K, Park CK. Optic disc torsion direction predicts the location of glaucomatous damage in normal-tension glaucoma patients with myopia. Ophthalmology 2012;119:1844-51.

22. Lee KS, Lee JR, Kook MS. Optic disc torsion presenting as unilateral glaucomatous-appearing visual field defect in young myopic Korean eyes. Ophthalmology 2014;121:1013-9.

23. Choi JA, Park HY, Shin HY, Park CK. Optic disc tilt direction determines the location of initial glaucomatous damage. Invest Ophthalmol Vis Sci 2014;55:4991-8.

24. Lee EJ, Kim TW, Kim JA, Kim JA. Central visual field damage and parapapillary choroidal microvasculature dropout in primary open-angle glaucoma. Ophthalmology 2018;125:588-96.

25. Lee EJ, Kim TW, Kim JA, et al. Elucidation of the strongest factors influencing rapid retinal nerve fiber layer thinning in glaucoma. Invest Ophthalmol Vis Sci 2019;60:334351.

26. Park HL, Kim JW, Park CK. Choroidal microvasculature dropout is associated with progressive retinal nerve fiber layer thinning in glaucoma with disc hemorrhage. Ophthalmology 2018;125:1003-13.

27. Kwon JM, Weinreb RN, Zangwill LM, Suh MH. Parapapillary deep-layer microvasculature dropout and visual field progression in glaucoma. Am J Ophthalmol 2019;200:65-75.

28. Kim JA, Lee EJ, Kim TW. Evaluation of parapapillary choroidal microvasculature dropout and progressive retinal nerve fiber layer thinning in patients with glaucoma. JAMA Ophthalmol 2019;137:810-6.

29. Lee EJ, Kim JA, Kim TW. Influence of choroidal microvasculature dropout on the rate of glaucomatous progression: a prospective study. Ophthalmol Glaucoma 2020;3:2531.

30. Ohno-Matsui K, Shimada N, Yasuzumi K, et al. Long-term development of significant visual field defects in highly myopic eyes. Am J Ophthalmol 2011;152:256-65. 